\title{
Análise de Limitações do Canal de Retorno em Sistemas OFDM Baseados em Códigos de Dispersão Linear
}

\author{
Fernando M. L. Tavares e André N. Barreto
}

\begin{abstract}
Resumo-Códigos de dispersão linear (LDC) vêm sido propostos como um método promissor para a obtenção de diversidade espacial em sistemas OFDM com múltiplas antenas de transmissão. Entretanto, para que os ganhos potenciais deste método possam ser atingidos é necessário que seja selecionada a palavra de código mais adequada em cada situação, o que em geral depende da existência de um canal de retorno eficiente e confiável. Contudo, canais de retorno apresentam limitações inerentes, como erros e atrasos na sinalização. Além disso, a fim de se reduzir a quantidade de bits de sinalização, normalmente não são enviadas informações de retorno para todos os símbolos e subportadoras. Neste artigo serão analisadas criticamente estas limitações e, levando-nas em conta, os ganhos que podem ser atingidos na prática com LDC serão verificados.
\end{abstract}

Palavras-Chave-Códigos de dispersão linear, LDC, canal de retorno

Abstract-Linear dispersion codes (LDC) are a promising method for attaining spatial diversity in OFDM systems with multiple transmit antennas. However, in order for these potential gains to be realised we need to select the most adequate codeword in each situation, which in general depends on the existence of an efficient and reliable feedback channel. Nevertheless, feedback channels have inherent limitations, such as errors and signalling delays. Besides, in order to reduce the number of signalling bits, feedback information is usually not sent for every symbol and subcarrier. In this article we will critically analyse these limitations, and, taking those into account, we'll see the performance gains that can be reached in practice with $L D C$.

Keywords - Linear dispersion codes, LDC, feedback channel

\section{INTRODUÇÃO}

Sistemas de comunicação sem fio com Múltiplas Entradas e Múltiplas Saídas (MIMO) com canal de retorno podem ser utilizados tanto para aumentar a taxa de transmissão como para reduzir a taxa de erro do enlace, adaptando os esquemas de recepção e transmissão segundo as informações disponíveis sobre o canal [1]. As informações do canal estão, normalmente, disponíveis para o receptor por meio da estimação de tons piloto, por exemplo, mas não estão disponíveis no transmissor. O canal de retorno é utilizado para que essa informação possa ser utilizada também pelo transmissor. Várias técnicas foram propostas para a utilização do canal de retorno de forma eficiente [2] - [6], as quais se baseiam na definição de um conjunto otimizado de matrizes de pré-codificação. No canal de retorno, é necessário então apenas informar qual a matriz (ou palavra de código) mais adequada para transmissão, e não

Fernando M. L. Tavares e André N. Barreto, Instituto Nokia de Tecnologia - INdT, Brasília, Brasil, e-mail: fernando.tavares@indt.org.br; andre.barreto.indt.org.br toda a informação de estado do canal. Este tipo de estratégia é chamado genericamente de códigos de dispersão linear (LDC).

Porém, em geral, tais técnicas não foram analisadas em situações mais realistas, em que o canal de retorno possui limitações. Primeiramente, ainda que em sistemas LDC o canal de retorno transmita informações quantizadas do canal, ainda assim é inviável enviar no canal de retorno qual a palavra de código para cada símbolo ou, em sistemas OFDM, para cada subportadora. Desta forma a informação de retorno se refere usualmente a apenas uma amostra do canal. Em segundo lugar, canais de retorno são sujeitos a erros e a atrasos. Neste artigo a técnica proposta em [2] será analisada levando-se em conta estas limitações inerentes aos canais de retorno, e será verificado que os ganhos do LDC só podem realmente ser realizados em algumas situações.

Este artigo está organizado da seguinte maneira: na Seção II, apresentação dos Códigos de Dispersão Linear; na Seção III, introdução da probabilidade de erro por pares de tais códigos; na seção IV, apresentação dos sistemas com canal de retorno limitado; na seção V, apresentação e discussão dos resultados da análise do canal de retorno; por fim, a seção VI, a conclusão do artigo.

\section{Códigos De Dispersão LiNeAR}

Os códigos de dispersão linear (LDC) [7] formam um grupo especial de códigos de bloco espácio-temporais, os quais são formados por combinações lineares de matrizes nas dimensões espaço e tempo. Nessas matrizes, os símbolos que serão transmitidos são considerados parâmetros. Códigos bastante difundidos na literatura, como os códigos propostos em [8], [9] e [10], podem ser considerados casos específicos dos LDC.

Considerando-se um sistema com $M_{T x}$ antenas de transmissão e $M_{R x}$ antenas de recepção, em que o código de bloco $\mathbf{X}$, de dimensão $M_{T x} \times \tau$, deve ser enviado em $\tau$ instantes de tempo, a equação (1) apresenta como tal código será formado a partir da combinação linear das matrizes de dispersão linear $\mathbf{A}_{q}$ e $\mathbf{B}_{q}$, de dimensão $M_{T x} \times \tau$, com a parte real, $\alpha_{q}$, e a parte imaginária, $\beta_{q}$, dos $Q$ símbolos de informação escolhidos de uma constelação de energia média unitária.

$$
\mathbf{X}=\sum_{q=1}^{Q} \alpha_{q} \mathbf{A}_{q}+j \beta_{q} \mathbf{B}_{q}
$$

Desta forma, pode-se escrever a equação de transmissão sobre 
o canal MIMO como:

$$
\mathbf{Y}=\sqrt{\frac{\rho}{M_{T x}}} \mathbf{H X}+\mathbf{N}
$$

onde $\rho, \mathbf{Y}$ e $\mathbf{H}$ denotam, respectivamente, a SNR média em cada antena de recepção, o bloco de código recebido $\left(M_{R x} \times\right.$ $\tau)$ e a matriz de canal $\left(M_{T x} \times M_{R x}\right)$. A matriz $\mathbf{N}$, por sua vez, representa a matriz de ruído aditivo Gaussiano com média zero e variância unitária.

Em [7], também foram introduzidas as restrições de energia, que devem ser respeitadas pelas matrizes de dispersão linear $\mathbf{A}_{q}$ e $\mathbf{B}_{q}$, apresentadas em (3), (4) e (5), da menos restritiva para a mais restritiva.

$$
\begin{gathered}
\sum_{q=1}^{Q} \operatorname{Tr}\left(\mathbf{A}_{q}^{H} \mathbf{A}_{q}+\mathbf{B}_{q}^{H} \mathbf{B}_{q}\right)=2 \tau M_{T x} ; \\
\operatorname{Tr}\left(\mathbf{A}_{q}^{H} \mathbf{A}_{q}\right)=\operatorname{Tr}\left(\mathbf{B}_{q}^{H} \mathbf{B}_{q}\right)=\frac{\tau M_{T x}}{Q}, q=1, \ldots, Q ; \\
\mathbf{A}_{q}^{H} \mathbf{A}_{q}=\mathbf{B}_{q}^{H} \mathbf{B}_{q}=\frac{\tau}{Q} \mathbf{I}_{M_{T x}}, q=1, \ldots, Q ;
\end{gathered}
$$

Em (3) é garantido que a potência total do sinal transmitido X seja normalizada. A restrição (4) garante que a potência total seja dividida igualmente entre os $Q$ símbolos. Por fim, em (5), é garantido também que a energia dos $Q$ símbolos seja dispersa uniformemente pelas dimensões da matriz $\mathbf{X}$.

\section{Probabilidade de Erro Por Pares de um Código DE DISPERSÃO LINEAR}

Com o objetivo de encontrar uma expressão para a probabilidade de erro por pares (PEP) de um LDC, é possível reescrever a equação (2) de forma conveniente. Sejam $\mathcal{A}_{q}$ e $\mathcal{B}_{q}$ definidos por

$$
\mathcal{A}_{q} \triangleq\left[\begin{array}{cc}
\mathbf{A}_{R, q} & -\mathbf{A}_{I, q} \\
\mathbf{A}_{I, q} & \mathbf{A}_{R, q}
\end{array}\right] ; \mathcal{B}_{q} \triangleq\left[\begin{array}{cc}
-\mathbf{B}_{I, q} & -\mathbf{B}_{R, q} \\
\mathbf{B}_{R, q} & -\mathbf{B}_{I, q}
\end{array}\right]
$$

onde $\mathbf{A}_{R, q}, \mathbf{A}_{I, q}, \mathbf{B}_{R, q}, \mathbf{B}_{I, q}$ são as partes real e imaginária das matrizes $\mathbf{A}_{q}$ e $\mathbf{B}_{q}$, respectivamente. Seja também $\underline{\mathbf{h}}_{n}$ definido por

$$
\underline{\mathbf{h}}_{n} \triangleq\left[\begin{array}{c}
\mathbf{h}_{R, n} \\
\mathbf{h}_{I, n}
\end{array}\right]
$$

onde $n=1, \ldots, M_{R x}$.

A partir dessas definições, tem-se portanto,

$$
\underbrace{\left[\begin{array}{c}
\mathbf{y}_{R, 1} \\
\mathbf{y}_{I, 1} \\
\vdots \\
\mathbf{y}_{R, M_{R x}} \\
\mathbf{y}_{I, M_{R x}}
\end{array}\right]}_{\mathbf{y}}=\sqrt{\frac{\rho}{M_{T x}}} \mathcal{H} \underbrace{\left[\begin{array}{c}
\alpha_{1} \\
\beta_{1} \\
\vdots \\
\alpha_{Q} \\
\beta_{Q}
\end{array}\right]}_{\mathbf{x}}+\underbrace{\left[\begin{array}{c}
\mathbf{n}_{R, 1} \\
\mathbf{n}_{I, 1} \\
\vdots \\
\mathbf{n}_{R, M_{R x}} \\
\mathbf{n}_{I, M_{R x}}
\end{array}\right]}_{\mathbf{n}}
$$

onde $\mathcal{H}$, a matriz real de canal equivalente de dimensões $2 M_{R x} \tau \times 2 Q$, é dada por

$$
\mathcal{H}=\left[\begin{array}{ccccc}
\mathcal{A}_{1} \underline{\mathbf{h}}_{1} & \mathcal{B}_{1} \underline{\mathbf{h}}_{1} & \ldots & \mathcal{A}_{Q} \underline{\mathbf{h}}_{1} & \mathcal{B}_{Q} \underline{\mathbf{h}}_{1} \\
\vdots & \vdots & \ddots & \vdots & \vdots \\
\mathcal{A}_{1} \underline{\mathbf{h}}_{M_{R x}} & \mathcal{B}_{1} \underline{\mathbf{h}}_{M_{R x}} & \ldots & \mathcal{A}_{Q} \underline{\mathbf{h}}_{M_{R x}} & \mathcal{B}_{Q} \underline{\mathbf{h}}_{M_{R x}}
\end{array}\right]
$$

Assim, pode-se definir a PEP de um LDC como a probabilidade de que um conjunto de símbolos e diferente de $\mathrm{x}$ seja recebido, dado que um conjunto de símbolos $\mathrm{x}$ foi enviado; considerando-se que essa probabilidade esteja condicionada à matriz de canal equivalente $\mathcal{H}$, em que $\gamma_{0}=\frac{\rho}{M_{T x}}$.

$$
P(\mathbf{x} \rightarrow \mathbf{e} \mid \mathcal{H})=P\left(\left\|\mathbf{y}-\gamma_{0} \mathcal{H} \mathbf{e}\right\|^{2}<\left\|\mathbf{y}-\gamma_{0} \mathcal{H} \mathbf{x}\right\|^{2}\right)
$$

Em [11], foi demonstrado que (11) pode ser utilizada como limite superior para (10).

$$
P(\mathbf{x} \rightarrow \mathbf{e} \mid \mathcal{H}) \leq\left(-\|\mathcal{H}(\mathbf{x}-\mathbf{e})\|^{2} \frac{\gamma_{0}^{2} M_{T x}}{4}\right)
$$

Entretanto, a inequação em (11) depende da constelação de símbolos utilizada. Para facilitar a análise dos LDC, foi proposta em [7] uma nova forma de calcular o limite superior para a PEP. Essa fórmula é apresentada em (12).

$$
P_{e}(\mathcal{H}) \leq \frac{1}{2} \operatorname{det}\left(\left[I+\frac{\gamma_{0}^{2}}{2} \mathcal{H}^{T} \mathcal{H}\right]^{-\frac{1}{2}}\right)
$$

\section{Sistemas com Canal de Retorno Limitado}

Como dito anteriormente, sistemas MIMO com canal de retorno permitem que a informação disponível sobre o canal no receptor possa ser enviada para o transmissor, de forma que ele a utilize para selecionar o melhor modo de transmissão dentre os disponíveis. Nota-se que esse tipo de sistema possui canal de retorno limitado quando tal canal permite o envio de apenas alguns poucos bits de informação.

Sistemas como esse foram estudados em [2] - [6], e em [2] foi proposto o uso de LDC. Nesse caso, partindo de um conjunto de LDC, o receptor, baseado no critério de probabilidade de erro por pares, enviará apenas o índice $i_{\text {opt }}$ do código LDC ótimo que deverá ser utilizado pelo transmissor. Dessa forma, é possível projetar sistemas baseados em LDC que conseguem extrair toda a diversidade do canal. Assim, serão enviados $n_{\text {bits }}$ suficientes para identificar $2_{n_{b i t s}}$ LDC diferentes que compõem o conjunto.

$$
i_{\text {opt }}=\arg \min _{i} P_{e}(\mathcal{H}(\{\mathbf{A}(i), \mathbf{B}(i)\}, \mathbf{H}))
$$

Com isso, um algoritmo iterativo poderá, a partir de um conjunto de LDCs pertencentes a um espaço de busca discreto predeterminado $\Omega$, buscar um conjunto ótimo $\Omega_{\text {opt }}$ de $2^{n^{\text {bits }}}$ LDC, o qual minimiza a PEP média do sistema, considerandose que o melhor LDC do conjunto será escolhido para cada amostra de canal utilizada pelo algoritmo, como em (14).

$$
\overline{P_{e}}(\mathcal{H})=E_{\mathbf{H}} \min _{\{\mathbf{A}, \mathbf{B}\} \in \Omega_{o p t}} P_{e}(\mathcal{H}(\{\mathbf{A}, \mathbf{B}\}, \mathbf{H}))
$$

\section{Limitações do CANAL DE RETORNO}

Além da limitação da quantidade de informação que pode ser enviada por meio do canal de retorno, outras são comuns em sistemas reais de comunicação. Entre as diversas limitações podem-se elencar o atraso no canal de retorno, a transmissão periódica de dados por esse canal e o agrupamento de subportadoras em grupos. Além disso, sempre existe a possibilidade de que haja erros no canal de retorno, o que também deve ser levado em conta. De forma a estudar cada uma dessas 


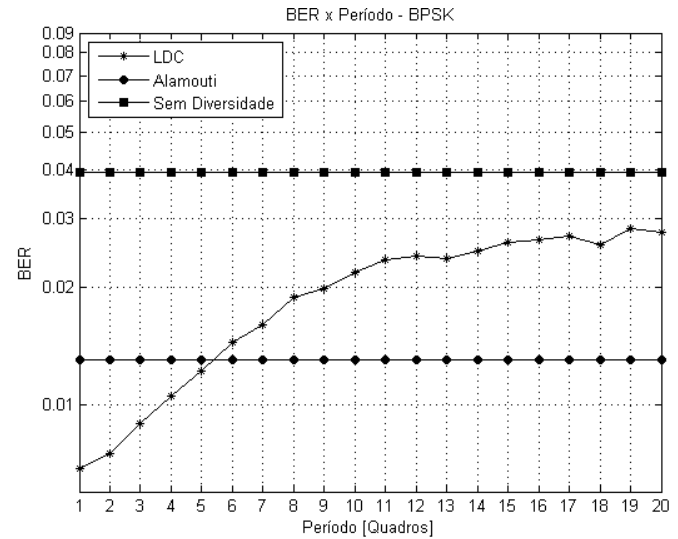

(a) Taxa de erro (BER) com período de sinalização variável - BPSK

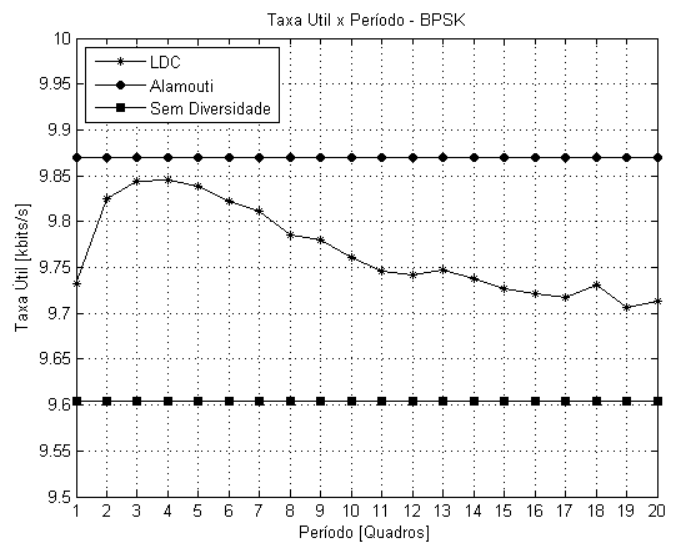

(c) Taxa útil $R$ com período de sinalização variável - BPSK

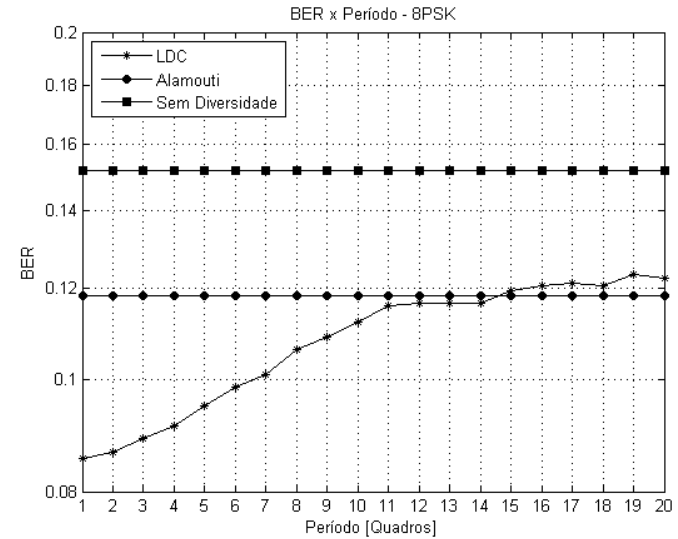

(b) Taxa de erro (BER) com período de sinalização variável - 8PSK

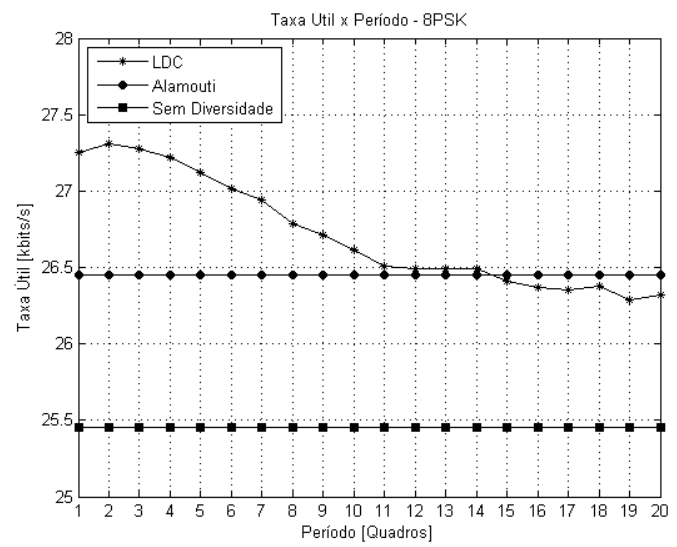

(d) Taxa útil $R$ com período de sinalização variável - 8PSK

Fig. 1. Resultados com período de sinalização variável.

limitações, será considerado um conjunto de LDC proposto em [2], apresentado em (15), na forma das matrizes $\mathbf{A}$ e $\mathbf{B}$, de dimensões $M_{T x}=2 \times \tau=1$ e apenas $Q=1$ símbolo por bloco.

$$
\mathbf{A}_{1}(1)=\mathbf{B}_{1}(1)=\left[\begin{array}{l}
1 \\
1
\end{array}\right], \quad \mathbf{A}_{1}(2)=\mathbf{B}_{1}(2)=\left[\begin{array}{c}
1 \\
-1
\end{array}\right]
$$

Com o intuito de avaliar o desempenho desse esquema, obtevese um vetor de canal OFDM na freqüência de $2.5 \mathrm{GHz}$, com $10 \mathrm{MHz}$ de banda, $N_{c}=1024$ portadoras e velocidade do móvel de $3 \mathrm{~km} / \mathrm{h}$, utilizando-se o modelo de Jakes, no qual os multipercursos e o efeito Doppler foram considerados. As antenas são consideradas descorrelatadas. Considerou-se também que o sistema possui apenas $M_{R x}=1$ antena de recepção. A transmissão é feita por quadros de $N_{s}=50$ símbolos OFDM, que são transmitidos a cada $T=5 \mathrm{~ms}$.

Serão avaliadas para cada caso, por meio de simulações computacionais, a taxa de erro de bits (BER) e a taxa de transmissão útil $R$ em regime de alta SNR, cujo valor será $20 \mathrm{~dB}$, onde o LDC apresenta maiores ganhos. Considerando um sistema duplex, em que o canal em cada sentido é o canal de retorno do seu par, pode-se calcular como

$$
R=\frac{\left(N_{s} N_{c} \log _{2} M-n^{b i t s}\right) \times(1-B E R)}{T} \mathrm{bits} / \mathrm{s},
$$

onde $M$ denota o número de símbolos da constelação em uso, M-PSK ou M-QAM.

\section{A. Transmissão Periódica de Dados}

Considerando-se um sistema no qual o canal de retorno é utilizado apenas no final de $N_{p}$ quadros. O receptor selecionará $i_{\text {opt }}$, para cada portadora OFDM, segundo o critério em (13), considerando a condição de canal instantânea, ou seja, o transmissor apenas receberá $i_{\text {opt }}$ a cada $N_{p} \times T$ segundos.

Como esperado, pode ser observado nas figuras 1(a) (BPSK) e 1(b) (8PSK) que a taxa de erro de bit (BER) é degradada com o aumento de $N_{p}$. Verifica-se também que, no caso em BPSK é utilizado, se $N_{p}>5$ então a taxa de erro será pior que no esquema Alamouti [8]. Para o caso em que a constelação é 8PSK, a taxa de erro será pior se $N_{p}>15$. Pode-se, além disso, perceber que com o aumento de $N_{p}$ a taxa com que a BER aumenta é reduzida, o que indica que a BER tenderá a estabilizar quando $N_{p}$ tender ao infinito. Isso se deve ao fato de que a informação enviada pelo canal de retorno torna-se imprecisa com o passar dos quadros, pois o canal utilizado é variante no tempo. Considerando-se que a frequência Doppler desse canal está em torno de $7 \mathrm{~Hz}$, o tempo de coerência será de $140 \mathrm{~ms}$, aproximadamente. Assim, quando $N_{p}$ se aproxima de 20 quadros, o sistema está operando em condições nas quais a correlação entre o estado do canal imediatamente anterior ao 


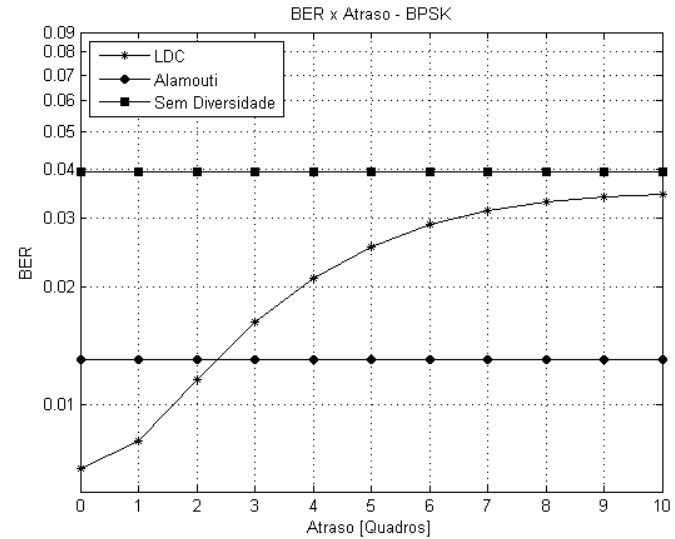

(a) Taxa de erro (BER) com atraso de sinalização variável BPSK

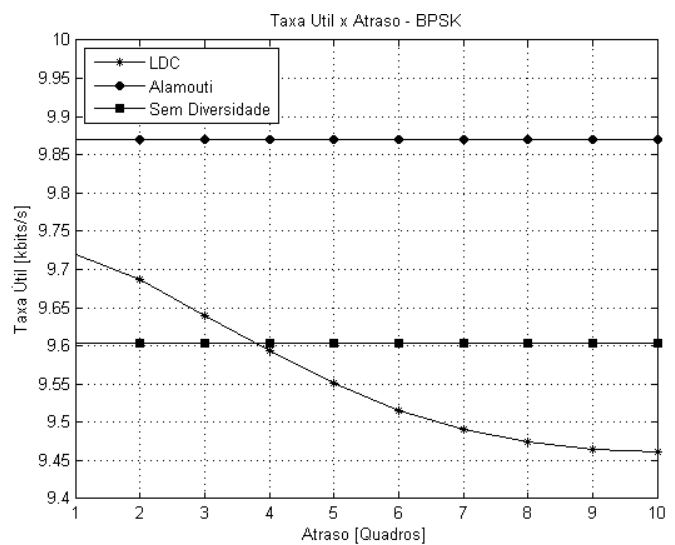

(c) Taxa útil $R$ com atraso de sinalização variável - BPSK

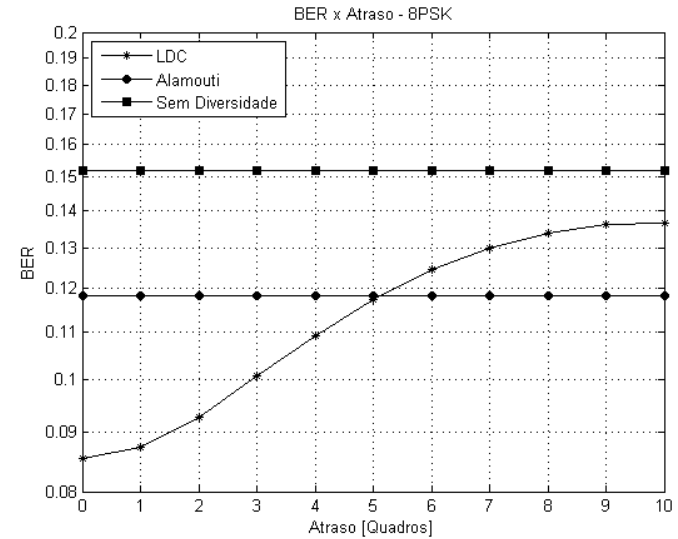

(b) Taxa de erro (BER) com atraso de sinalização variável 8 PSK

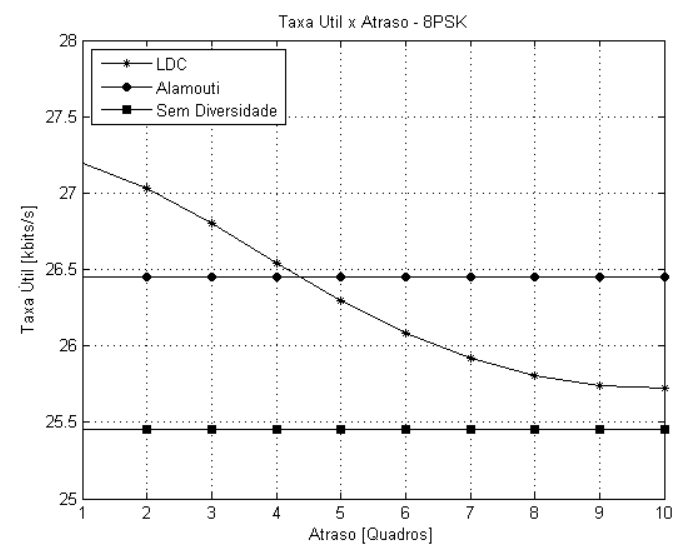

(d) Taxa útil $R$ com atraso de sinalização variável - 8PSK

Fig. 2. Resultados com atraso de sinalização variável.

próximo envio e o estado do canal considerado no momento do envio da informação pelo canal de retorno é muito pequena.

Nas figuras 1(a) e 1(b) são apresentados também o desempenho do esquema que utiliza o código Alamouti e do esquema sem diversidade espacial, em que apenas uma antena de transmissão e uma antena de recepção estão disponíveis. Vale ressaltar que o código Alamouti, utilizado como base de comparação neste estudo, é um caso particular de LDC.

Verifica-se, contudo, na figura 1(c) (BPSK) que a taxa útil $R$ apresenta um pico em $N_{p}=4$. Isto se deve ao aumento da taxa de erro com o aumento de $N_{p}$, mas o número de bits enviados pelo canal de retorno diminui, o que diminui a taxa utilizada para sinalização, resultando em maior taxa útil. Podese observar ainda que, apesar de apresentar taxa de erro melhor que o esquema Alamouti para $N_{p}<5$, o esquema LDC com constelação BPSK não oferece taxa útil melhor que o esquema Alamouti para nenhum valor de $N_{p}$. Por outro lado, ao avaliar a taxa útil para o caso com constelação 8PSK, observa-se que a taxa útil é maior que no caso do esquema Alamouti para $N_{p}<10$. Assim, pode-se concluir, com o auxílio da equação (16), que, com o aumento da ordem da modulação utilizada, os ganhos serão mais significativos.

\section{B. Atraso no Canal de Retorno}

Considerando-se o mesmo sistema do item anterior, com $N_{p}=1$, no qual exista um atraso de $N_{d}$ quadros. Nesse caso, o receptor selecionará e enviará $i_{\text {opt }}$ a cada quadro, mas o transmissor receberá essa informação apenas $D \times 5$ ms mais tarde.

Nas figuras 2(a) (BPSK) e 2(b) (8PSK), pode-se notar que a taxa de erro de bits (BER) é degradada com o aumento de $N_{d}$ em ambos os casos. Utilizando BPSK, a taxa de erro será melhor que a do esquema Alamouti se $N_{d}<2$. Caso 8PSK seja utilizado, a taxa de erro será melhor até $N_{d}<5$.

Novamente, pode-se notar que a curva da taxa de erro tende a um valor constante com o aumento de $N_{d}$, e a explicação dada no item anterior também é válida. Com o aumento do atraso, a informação enviada pelo canal será utilizada pelo receptor apenas $N_{d} \times T$ segundos, e com o aumento do atraso, a correlação será pequena entre o estado do canal utilizado para a seleção do LDC ótimo e o estado do canal no instante em que a informação será utilizada pelo transmissor.

Além disso, ao verificar a taxa útil $R$ para ambos os casos, nas figuras 2(c) (BPSK) e 2(d) (8PSK), percebe-se que, caso a constelação BPSK seja utilizada, a taxa útil $R$ do esquema LDC nunca será melhor que a taxa útil do esquema Alamouti. Para a constelação 8PSK, será interessante utilizar o esquema LDC caso o $N_{d}<5$. 


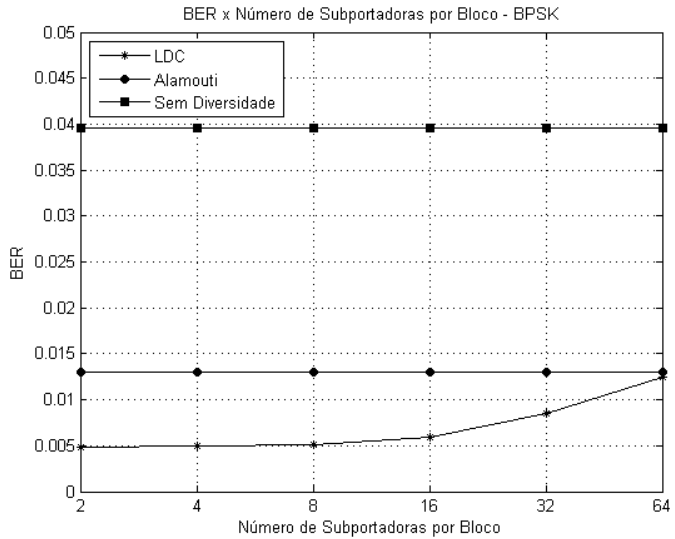

(a) Taxa de erro (BER) com número de subportadoras por grupo variável - BPSK

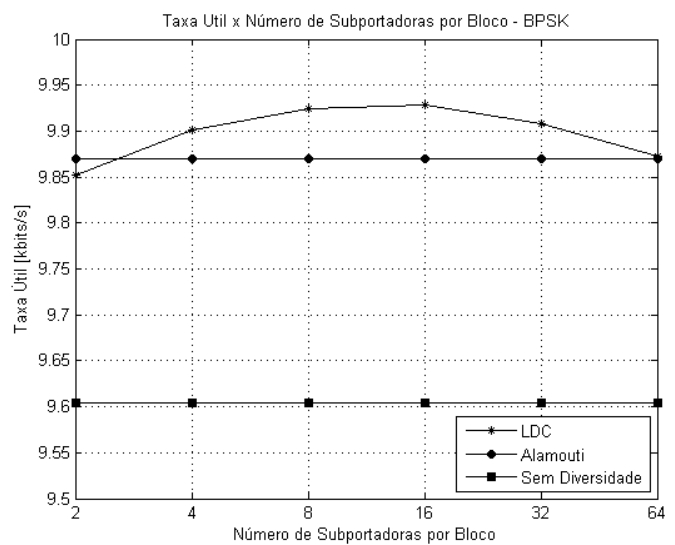

(c) Taxa útil $R$ com número de subportadoras por grupo variável - BPSK

Fig. 3. Resultados com número de subportadoras por grupo variável.

\section{Agrupamento de Subportadoras}

Considerando-se o mesmo sistema do item anterior, com $N_{d}=0$ e $N_{p}=1$, no qual as subportadoras OFDM foram agrupadas em grupos. Neste caso, o índice $i_{\text {opt }}$ enviado será utilizado pelo transmissor para as $N_{b}$ subportadoras do grupos.

Nota-se, nas figuras 3(a) (BPSK) e 3(b) (8PSK), que a taxa de erro de bits (BER) aumenta com o crescimento do número de subportadoras no grupo. Entretanto, este aumento passa a ser significativo apenas quando $N_{b}>8$. Ao verificar a taxa útil $R$, nas figuras 3(c) (BPSK) e 3(d) (8PSK), nota-se também que existe um ponto de máximo em torno de $N_{b}=8$ e $N_{b}=16$. Pode-se afirmar, portanto, que um número ideal de subportadoras por grupo pode ser obtido. Entretanto, as figuras indicam que o agrupamento de subportadoras não é a limitação que causa o maior impacto neste tipo de sistema.

\section{Erros no Canal de Retorno}

Considerando-se agora $N_{d}=0$ e $N_{p}=1$, no qual existe a probabilidade $P_{f}$ de que haja erro no envio da informação pelo canal de retorno. Assim, existe a possibilidade de que o transmissor utilize uma informação errada para selecionar o LDC que utilizará para transmitir no próximo bloco.

Nas figuras 4(a) (BPSK) e 4(b) (8PSK), nota-se que o ganho de um esquema com LDC é reduzido quando a taxa de erro

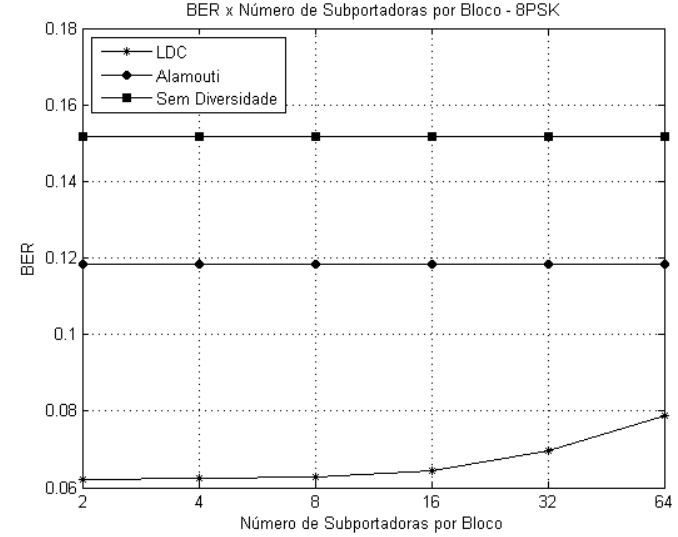

(b) Taxa de erro (BER) com número de subportadoras por grupo variável - 8PSK

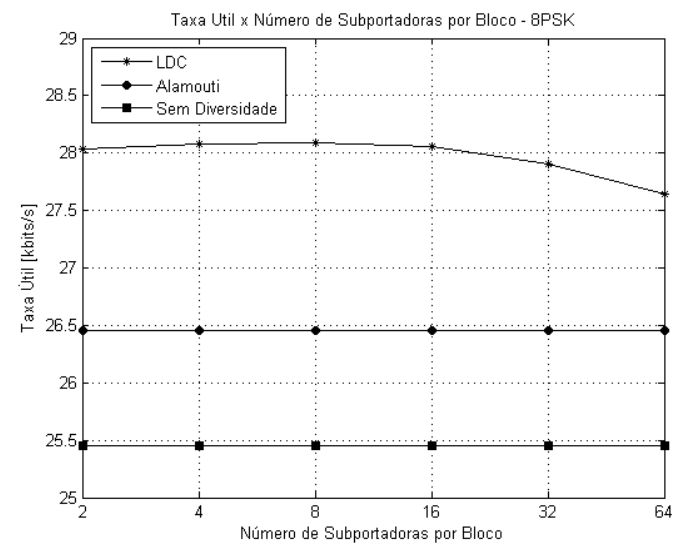

(d) Taxa útil $R$ com número de subportadoras por grupo variável - 8PSK

do canal de retorno aumenta. Nota-se ainda que, quando a constelação BPSK é utilizada, caso $P_{f}>20 \%$, a taxa de erro será melhor que utilizando o código LDC. Caso o 8PSK seja utilizado, a taxa de erro do esquema que utiliza o código LDC será a melhor apenas quando $P_{f}<50 \%$.

Verificando a taxa útil $R$, observa-se que caso o BPSK seja utilizado, não é recomendado o uso de um LDC. Caso o 8PSK seja utilizado, este será melhor que o código Alamouti quando a probabilidade de erro no canal de retorno for menor que $40 \%$, aproximadamente.

É importante ressaltar, no entanto, que, em todos os casos analisados, foi considerado que o canal de retorno não utiliza codificação para a transmissão da informação necessária para a seleção do LDC ótimo. Em sistemas reais, sejam legados, sejam em desenvolvimento, o canal de retorno é codificado a altas taxas para evitar que ocorram erros. Dessa forma, para cada bit transmitido pelo canal de retorno, em geral, dezenas de bits serão transmitidos para garantir que cada bit seja recebido.

Assim, a limitação imposta pelos erros no canal de retorno pode ser removida, mas o custo de sinalização associado fará com que os ganhos do LDC, mesmo em regime de alta SNR, sejam reduzidos até um ponto onde, provavelmente, será mais interessante utilizar um sistema no qual não seja necessário o 
XXVII SIMPÓSIO BRASILEIRO DE TELECOMUNICAÇÕES - SBrT 2009, DE 29 DE SETEMBRO A 2 DE OUTUBRO DE 2009, BLUMENAU, SC

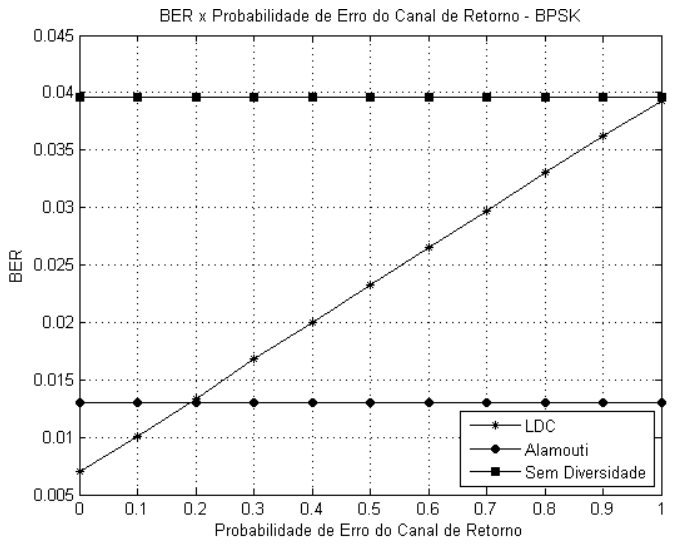

(a) Taxa de erro (BER) com erro de sinalização variável BPSK

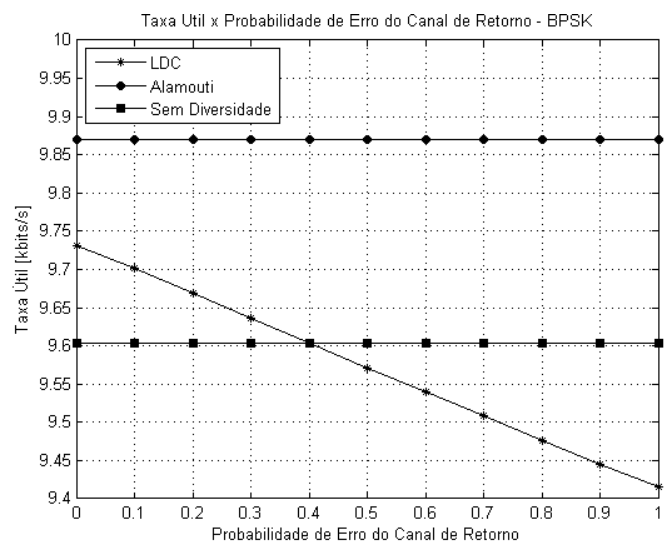

(c) Taxa útil $R$ com erro de sinalização variável - BPSK

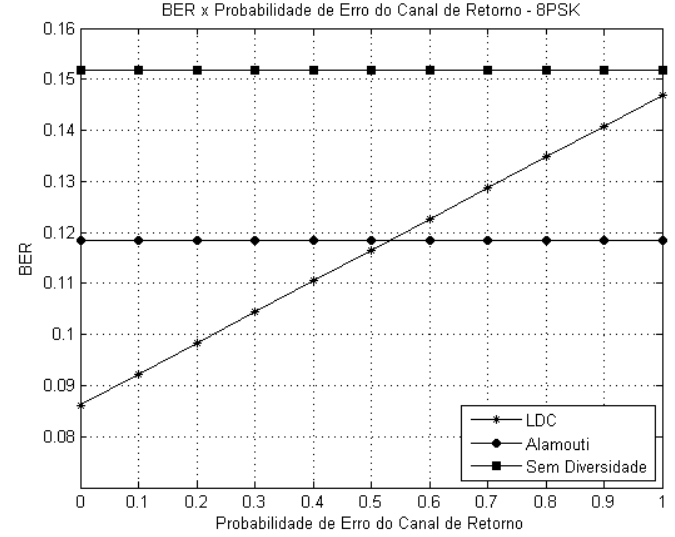

(b) Taxa de erro (BER) com erro de sinalização variável 8PSK

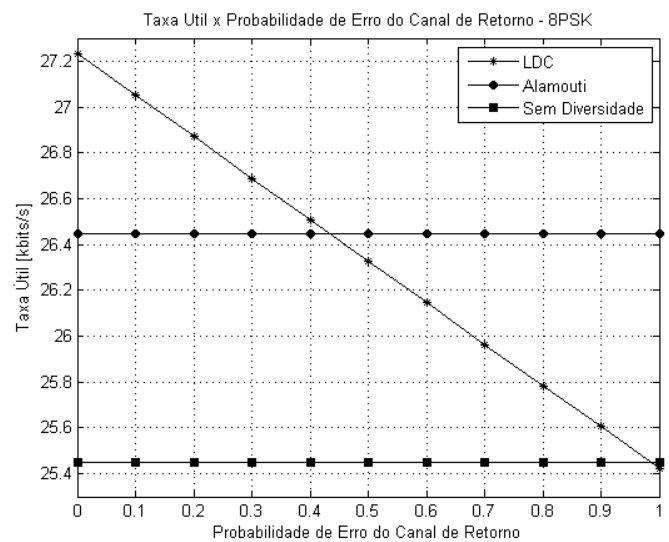

(d) Taxa útil $R$ com erro de sinalização variável - 8PSK

Fig. 4. Resultados com erro de sinalização variável.

uso do canal de retorno.

\section{CONCLUSÃo}

Alguns autores defendem o emprego de sistemas MIMO com canal de retorno com o intuito de extrair a diversidade espacial, utilizando como argumento a possibilidade de redução da taxa de erro. Neste artigo, ao analisar um esquema baseado em códigos de dispersão linear em situações nas quais seu canal de retorno é submetido a limitações comuns a sistemas reais, observou-se que a viabilidade da utilização de tal esquema está condicionada a características da sinalização, como o período, o atraso e o número de subportadoras por grupo, e a fatores como a constelação utilizada.

Ademais, os resultados obtidos demonstram a necessidade de considerar a taxa útil do sistema, uma vez que, na prática, essa taxa sofre redução devido à sinalização frequente. Assim, caso seja utilizada a codificação com altas taxas no canal de retorno, como é comum em sistemas reais, o emprego de esquemas que dependem do canal de retorno, como o esquema baseado em LDC e seus similares, não é recomendado.

\section{AgRADECIMENTOS}

Este trabalho foi parcialmente financiado pelo CNPq através do projeto WiPA, Processo 554047/2006-3.

\section{REFERÊNCIAS}

[1] A. Paulraj, R. Nabar e D. Gore, Introduction to Space-Time Wireless Communications. Cambridge Univ. Press, Maio 2003.

[2] R. Machado, B. F. Uchoa-Filho e T. M. Duman, Linear Dispersion Codes for MIMO Channels with Limited Feedback. IEEE WCNC 2008, pp. 199-204, Abril 2008

[3] D. J. Love, R. H. Heath e T. Strohmer, Grassmannian Beamforming for Multiple-Input Multiple-Output Wireless Systems. IEEE Trans. on Inf. Theory, v. 49, pp. 2735-2747, Outubro 2003.

[4] N. Jindal, MIMO Broadcast Channels with Finite Rate Feedback. IEEE Trans. on Inf. Theory, v. 52, pp. 5045-5060, Novembro 2006.

[5] Y. Seung-Hyeon, K. Jae-Yun e L. Yong-Hwan, Transmit Beamforming with Reduced Feedback Information in OFDM Based Wireless Systems. IEEE VTC Spring 2008, pp. 983-987, Maio 2008.

[6] S. Sorrentino e L. Moretti, Efficient Precoder Quantization for TimeVarying MIMO Wireless Channels. IEEE VTC Spring 2008, pp. 787791, Maio 2008.

[7] B. Hassibi e B. M. Hochwald, High-rate codes that are linear in space and time. IEEE Trans. on Inf. Theory, v. 48, pp. 1804-1824, Julho 2002.

[8] S.M. Alamouti, A simple transmit diversity technique for wireless communications. IEEE Journal on Selected Areas in Communications, v. 16, pp. 1451-1458, Outubro 1998.

[9] V. Tarokh, H. Jafarkhani e A.R. Calderbank, Space-time block codes from orthogonal designs. IEEE Transactions on Information Theory, v. 45, pp. 1456-1467, Julho 1999.

[10] R. Machado e B.F. Uchoa-Filho, Space-time block coding with hybrid transmit antenna/code selection. IEEE International Conference on Communications 2004, v. 2, pp. 819-822, Junho 2004.

[11] V. Tarokh, N. Seshadri e A. R. Calderbank, Space-time codes for high data rate wireless communications: Performance criterion and code construction. IEEE Trans. Inf. Theory, v. 44, pp. 744-765, Março 1998. 